\begin{tabular}{l|l|ll|lll|ll}
\hline R. de Econometria & Rio de Janeiro & v. VII nọ & 1 & p. & $55-82$ & abril & 1984 \\
\hline
\end{tabular}

A DEMANDA DE SERVICO DE TELEX NACIONAL: UM ESTUdo ECONOMÉTRICO*

Helio Lechuga Arteiro**

RESUMO

o objetivo deste artigo é desenvolver uma especificação de um modelo econométrico para o melhor conhecimento das variáveis que determinam a demanda de serviços de Telex no Brasil.

* Este artigo è resultado de un trabalho apresentado em dezembro de 1986 na Es cola de Pós-Graduação em Economia da Fundação Getúlio Vargas.

** Economista da ärea de Preşos e Tarifas da Empresa Brasileira de Telecomunica cöes - EMBRATEL. 
ABSTRACT

This article aims to develop a specification of an econometric model in order to understand better the variables that determine the demand for Telex service in Brazil.

1. Introduction; 2. Theoretical model; 3. Empirical results;

4. Concluding remarks. 
1. INTRODUCAO

Apesar do importante papel desempenhado pelos serviços públi cos de infraestrutura econômica no processo de desenvolvimento do Pais, muito pouco se tem escrito sobre a demanda destes servicosno Brasil. No setor das telecomunicações, por exemplo, podemos citar - serviço telex que nos últimos anos atingiu um alto grau de penetração no território nacional, interligando hoje mais de 70000 ter minais, dispersos por cerca de 1800 localidades.

Neste artigo serão apresentadas algumas contribuições para o melhor conhecimento das variáveis que determinam a demanda de serviços de telex no Brasil, obtidas atravēs da especificação de um modelo econométrico com base na teoria econômica. Nos EUA, os estu dos para determinação de curvas de demanda na área de telecomunica ções,

existência de significativas diferenças entre o perfil da demanda de telefonia e telex, que impede o aproveitamento de metodologiajã existente, o presente artigo dedica-se a desenvolver nova metodolo gia, adaptada às características da exploração deste serviço no Brasil.

Por suas características, o Telex destina-se basicamente ao atendimento do mercado empresarial, não havendo casos de usuários residenciais entre seus assinantes. Deste modo, tal serviço atua na qualidade de insumo, participando dos processos produtivos das empresas usuárias. Por esta razão, è desenvolvida uma análise com base na teoria existente para o tratamento do mercado de fatores de produção.

Restam ainda dois pontos importantes a considerar antes de ser iniciada a especificação do modelo: o primeiro è que a demanda pe- 
la instalação de linhas de acesso e por terminais teleimpressores; o segundo consiste no fato de que o processo de ajustamento entre a quantidade desejada de linhas de acesso e a existente nāo é imediata, devido à característica de longa duraçāo dos projetos para expansão da capacidade da planta. A ocorrência de um desequilíbrio entre a quantidade desejada de linhas de acesso e a existente impe de que a quantidade total de mensagens que seus usuários desejam transmitir seja atingida.

De posse de tais informações poderá ser dado início à apresen taçāo do modelo teórico, conforme veremos a seguir.

2. MODELO TEORICO

\subsection{A Demanda pelo Servico}

Considerando que os serviços de telecomunicações são utilizá dos pelas empresas como insumos em seus processos produtivos, a de manda pelo Serviço Telex deve-se à necessidade destas firmas em transmitir e receber mensagens na forma de texto. Com base na teoria existente para o tratamento do mercado de fatores de produção, sabe-se que para realização de seus processos produtivos, as empre sas utilizam uma relaçāo técnica que combina uma certa dotaçāo de insumos, de forma a maximizar o produto final.

Seja $Q$ a quantidade a ser produzida, $e x_{1}, x_{2}, \ldots, x_{n}$, a quan tidade dos $\mathrm{n}$ insumos utilizados. A função de produçāo resultante pode ser expressa por:

$$
Q=Q\left(x_{1}, x_{2}, \ldots, x_{n}\right)
$$

Considerando que os empresários procuram produzir ao menor cus to possivel, temos que a quantidade demandada de um determinado fa tor de produção, $x_{1}$, é funçāo da quantidade $Q$ a ser produzida, além, do preço de $x_{1}$ e do preço dos demais fatores complementares ou substitutos de $x_{1}$. 


$$
x_{1}=x_{1}\left(r_{1}, r_{2}, \ldots, r_{n}, Q\right)
$$

sendo $r_{i}=$ o preço do fator $i$, para $i=1,2, \ldots, n$.

Seja dd a demanda desejada por mensagens telex, sendo esta uma funçāo do custo unitārio da mensagem, do custo de serviços subs titutos e da quantidade desejada de bens e serviços finais a serem produzidos pelas empresas.

$$
d d=g(P m, P s, Q)
$$

onde: $\quad \mathrm{Pm}=$ Preço da mensagem telex

Ps = Preços de serviços substitutos

$\mathrm{Q}=$ Quantidade desejada a ser produzida

A variável preço da mensagem engloba também os custos de assí natura e do terminal teleimpressor. Com base na Teoria Econômica espera-se que o sinal do coeficiente desta variável seja negativo, enquanto que o sinal dos coeficientes das duas outras seja positivo.

Como a demanda por mensagens origina as demandas pelos respec tivos meios que possibilitam tal transmissāo, ou seja, os teleimpressores e linhas de acesso, a demanda desejada pelo estoque de linhas de acesso, KD, poderá ser expressa pelo total das inscrições de usuārios para o serviço telex, menos as desistências. A de manda desejada por linhas de acesso englobará, portanto, as inscri ções atendidas e as pendentes.

o modelo considera as demandas por teleimpressores e meios $f_{\underline{i}}$ sicos como aproximadamente iguais, uma vez que, salvo em raras exceções, nāo se justifica a existência de um teleimpressor, sem a disponibilidade de uma linha de acesso, permitindo que as conside rações apresentadas para uma, sejam tambēm vālidas para a outra.

Tendo em vista que a procura por linhas de acesso é proporcio nal à demanda desejada por mensagens, temos que:

$$
K D=j \times g(P m, P s, Q)
$$


E importante observar que $j$ representa $o$ inverso da variável número médio de mensagens transmitidas por terminal, dm, sendo esta última expressa pela seguinte função:

$\mathrm{din}=2(\mathrm{Pm}, \mathrm{Ps}, \mathrm{Q}, \mathrm{KE})$

Os sinais dos coeficientes das variāveis explicativas da função apresentada acima, são os mesmos da função demanda desejada por mensagens, dd, com exceção obviamente de KE que representa o estoque existente de linhas de acesso. Nesta função o coeficiente de $\mathrm{KE}$ pode assumir um sinal indeterminado uma vez que, por um lado, quanto maior o tamanho da Rede Pública, maiores serão as possibilị dades de trāfego para um determinado terminal, enquanto que, por outro, quanto maior o número de terminais em um mesmo escritório, menor è a concentração das mensagens transmitidas por terminal, o que faria o tráfego médio cair. Deste modo, KD pode ser expresso por:

$\mathrm{KD}=\frac{\mathrm{g}(\mathrm{Pm}, \mathrm{Ps}, \mathrm{Q})}{2(\mathrm{Pm}, \mathrm{Ps}, \mathrm{Q}, \mathrm{KE})}$

Em termos de uma única função, teremos:

$K D=f(P m, P s, Q, K E)$

Como pode-se observar, os coeficientes das variāveis $\mathrm{Pm}$, Ps e @ assumem sinais indeterminados, enquanto que o coeficiente de $\mathrm{KE}$ assume um valor inverso ao apresentado na equação de número médio de mensagens por terminal.

Demonstraremos no próximo item que KE é uma funçāo de KD. Sen do assim, teremos:

$$
K D=f(P m, P s, Q, m(K D))
$$

Pode-se esperar que a derivada de $\mathrm{KD}$ em relação a $\mathrm{KE}$ seja menor do que um, neste caso a função KD será expressa apenas em termos de Pm, Ps e 2 , e os sinais dos coeficientes destas variáveis nāo se alteram. 


$$
K D=f(P m, P s, Q)
$$

Por motivos óbvios, suporemos que $K E=K D$, neste caso teremos que $\mathrm{dr}=\mathrm{dd}$. A demanda realizada de mensagens, dr, que è expressa pelo total de pulsos gerados na Rede pública em um determinado mēs, deve-se à impossibilidade de efetivação da quantidade desejada de mensagens, tendo em vista a restrição quanto ao estoque ativado de teleimpressores e de linhas de acesso. A demanda realizada de mensagens será expressa então por:

$$
d r=d d-\Phi(K D-K E)
$$

Pela equação anterior, pode-se concluir que quando $\mathrm{KD}=\mathrm{KE}$, te remos então $d r=d d$. Desenvolvendo tal equação, temos:

$$
\begin{aligned}
& d r=d d-\Phi K D+\Phi K E \\
& d r=g(P I n, P s, Q)-\Phi f(P A n, P s, Q)+\Phi K E
\end{aligned}
$$

Sendo assim, a demanda realizada por mensagens será dada pela seguinte função:

$$
d r=h(P m, P s, Q, K E)
$$

\subsection{Estoque Existente de Linhas de Acesso}

A EMBRATEL como empresa estatal, vinculada ao Ministério das Comunicaçōes, torna-se muito sensivel a objetivos governamentais de natureza social, não se constituindo a rentabilidade em critério único em suas decisōes de investimentos. Os níveis de atendimento à demanda e sua abrangência geográfica, muitas vezes, são determinantes maiores para a ampliação, até mesmo de serviços cuja rentabilidade é insatisfatória.

Outrossim, pode-se afirmar que o processo de ajustamento entre o estoque desejado de linhas de acesso e o existente não costú ma ser imediato, uma vez que no caso de ausência de meios físicos, os prazos de maturação de projetos para expansão da capacidade da 
planta são freqthentemente de longa duração. Deste modo, para a at vação de um novo terminal, è necessārio, primeiramente, a existência de capacidade de comunicação nas centrais públicas, e de concentradores que permitam a maximização da utilização destas centrais, alēm da existência de linhas de acesso que interliguem um teleimpressor de usuário a uma Central pública. Havendo a necessidade de instalação de uma nova central de comutação, o prazo de ativação de um novo usuário atinge em média de dois a três anos. Havendo, também, a necessidade da instalação de concentrador, são necessārios aproximadamente 12 meses. No caso da ativação de uma linha de acesso, pode-se tornar necessārio um período de até seis meses.

Nas situaçōes em que os meios físicos necessārios estiverem disponiveis, o atendimento a uma determinada inscrição poderá ser feito quase que imediatamente.

Devido às características da exploração do serviço, realizare mos alguns testes sobre a adaptabilidade do modelo desenvolvidopor Jorgenson (1965), para explicação da estrutura temporal do processo de investimento.

Partiremos da hipótese de que no momento em que um certo usuá rio se inscreve para o serviço, ê iniciado um processo para sua ativação, cujo período de duração variarā em função da disponibilìdade de meios físicos. Além disso, sabe-se que a ocorrência de desistências de usuários anteriormente inscritos, mas não atendidos, pode representar a antecipação no atendimento de inscriçōes mais recentes. Neste caso o número de novos projetos de atendimento a usuários, iniciados no mês $t$, seria dado pelo total das inscriçōes no mês $t$, menos as desistências no próprio mês. Deste modo, teremos então que:

$$
K D_{t}-K D_{t-1}=I N_{t}
$$

onde: $\quad \mathrm{KD}_{\mathrm{t}}-\mathrm{KD}_{\mathrm{t}-1}=$ Variação líquida do total das inscriçōes no mês $t$.

$I N_{t}=0$ somatório dos novos projeots de atendimento às inscriçōes, iniciados no mês $t$. 
Seja $I E_{t}$ o total dos projetos de ativaçāo de novos usuários realizados no mês $t$, o que é igual ao número de ativaçōes efetuados no mês $t$.

$$
I E_{t}=K E_{t}-K E_{t-1}
$$

onde: $\quad \mathrm{KE}_{t}-\mathrm{KE}_{\mathrm{t}-1}=$ Variação física do estoque existente de terminais ativados.

Sabendo-se que apenas uma parcela dos projetos de atendimento iniciados no mês $t$, podem ser finalizados no mês $t$, enquanto que $u$ ma parcela dos projetos iniciados em ( $t-1)$, sāo atendidos no mês $t$, e assim sucessivamente. Temos entāo que:

$$
\begin{aligned}
I E_{t}= & U_{0} I N_{t}+U_{1} I N_{t-1}+U_{2} I N_{t-2}+\ldots+U_{n} I N_{t-1} \\
U_{k}= & \text { a proporção dos projetos iniciados em }(t-k) \text { e finaliza- } \\
& \text { dos no mès } t, \text { para } K=0,1,2, \ldots, n
\end{aligned}
$$

Em outras palavras o total dos terminais ativados no mês $t$, $I E_{t}$, é decorrente de uma parcela das inscriçōes líquidas realizadas nos meses $t, t-1, t-2, \ldots, t-n$.

Em nosso modelo, admitimos a hipótese de que todo projeto para atendimento a um novo usuário é concluído mais cedo ou mais tar de; estamos admitindo, também, que o atendimento às inscriçōes assume um comportamento regular. Deste modo, pode-se esperar que o somatório dos coeficientes desta equação seja igual à unidade.

$$
\mathrm{u}_{0}+\mathrm{u}_{1}+\mathrm{u}_{2} \cdots+\mathrm{u}_{\mathrm{n}}=1
$$

\subsection{Modelo Completo}

Pelo que foi apresentado até o momento, chegamos a um modelo composto por três equaçōes resultantes da funçāo de demanda realiza da por mensagens, da função de demanda desejada por linhas de aces so e da equação explicativa do fluxo de ativaçōes de novos terminais. 
Partimos do pressuposto da existência de relações não linea res entre as variáveis dependentes das equações de demanda e suas respectivas variāveis explicativas. Para simplificar os métodos de estimação, optamos por utilizar funções log-lineares na primeira e terceira equação do modelo, deste modo, a letra I introduzida na frente das variāveis destas duas equações representa o logarítmone periano destas variāveis. o modelo completo pode ser representado por:

$$
\begin{aligned}
& L d r_{t}=a_{0}+a_{1} L P m_{t}+a_{2} L P s_{t}+a_{3} L \Omega_{t}+a_{4} L K E_{t}+e_{t} \\
& K_{t}-K E_{t-1}=b_{1}\left(K D_{t}-K D_{t-1}\right)+\ldots+b_{n}\left(K D_{t-n}-K D_{t-n-1}\right)+S_{t} \\
& L K D_{t}=C_{0}+C_{1} L P m_{t}+C_{2} L P s_{t}+C_{3} L Q_{t}+V_{t} \\
& \text { Sendo assim, as variäveis endógenas do modelo são: } \\
& d r_{t}=\text { demanda realizada de mensagens } \\
& K E_{t}=\text { estoque ativado de terminais } \\
& K D_{t}=\text { estoque desejado de terminais }
\end{aligned}
$$

Pelo que se pode observar, estamos diante de um sistema recur sivo, uma vez que a terceira equação de nosso modelo possui apenas variāveis exógenas no conjunto de suas variáveis explicativas. Des te modo, o curso das influências segue apenas uma direção, ou seja, o resultado da terceira equação interfere na segunda, enquanto que esta por sua vez interfere no resultado da primeira equação. lém disto, para que o modelo seja recursivo, assumiremos que a matriz de variância e covariância seja diagonal.

Apresentaremos, a seguir, os resultados dos testes realizados com o modelo. 


\section{RESULTADOS EMPIRICOS}

\section{1 Demanda por Mensagens}

Partamos da primeira equação de nosso modelo, ou seja, a demanda realizada por mensagens, que em termos funcionais é:

$$
\mathrm{dr}=\mathrm{h}(\mathrm{P} \pi \mathrm{t}, \mathrm{Ps}, \mathrm{Q}, \mathrm{KE})
$$

onde: $\mathrm{dr}=$ demanda realizada de mensagens

$$
\mathrm{Pm}=\text { preço médio da mensagem telex }
$$$$
\text { Ps = preço de serviço substituto }
$$$$
Q=\text { nivel de atividade produtiva do usuário }
$$$$
\mathrm{KE}=\text { terminais ativados }
$$

E tomemos, para simplificar, os métodos de cálculo, o total de pulsos gerados na rede pública em um determinado mês, como uma medida representativa do número de mensagens transmitidas. Analisa remos a seguir as variáveis que participam na função de demanda rea lizada por mensagens.

\subsubsection{Preço Médio da Mensagem}

Para expressar a variāvel preço médio da mensagem, construímos um indice representativo do valor do pulso, incluindo em sua composição as despesas com equipamentos e meios necessārios à trans missão de mensagens. Alēm da tarifa média por pulso, utilizamostam bém o valor médio por pulso da tarifa de assinatura e do preço do aluguel ou aquisição de um teleimpressor.

No caso de aquisição de teleimpressor diretamente ao fabrican te, realizamos uma transformação do valor a vista, para uma série mensal, utilizando para isto uma taxa de depreciação linear de 0,838 ao mês, considerando o período de vida útil de 10 anos, alēm de uma taxa de juros real equivalente a 18 ao mês a titulo de remunera cão do custo oportunidade do capital investido. 
A equação do preço médio da mensagem è dada por:

$$
\mathrm{Pm}=\mathrm{PP}+\frac{\mathrm{PA}+(\alpha+\mathrm{PL})+(\beta \times(\mathrm{PT}+\mathrm{PM}))}{\mathrm{TRAM}}
$$

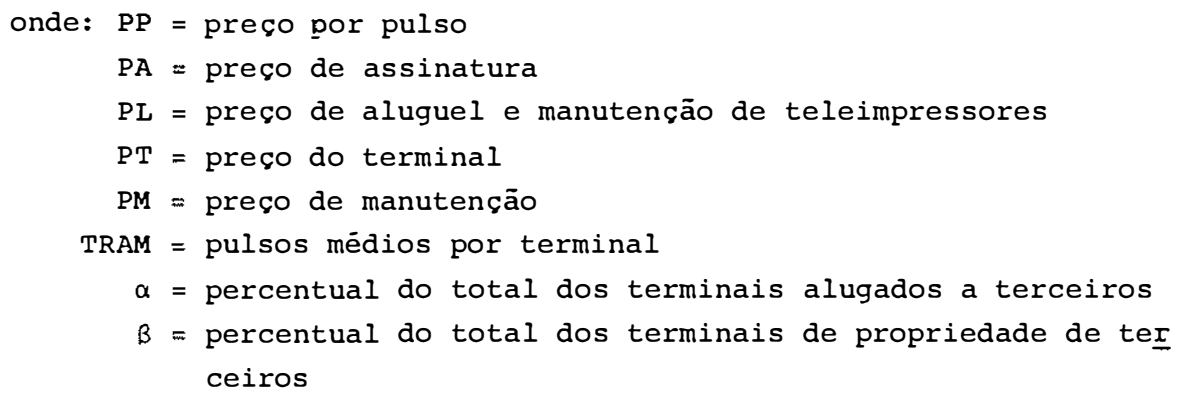

Pela formulação da equação de pulsos totais, estamos admitindo que o tráfego de mensagens gerados no periodo $t$ é explicado pelos preços em vigor no próprio período. Tal hipótese pressupõe que os usuários possuem um perfeito conhecimento sobre as tarifas vigentes no momento das transmissões, bem como de previsões sobre o total de mensagens a serem enviadas no periodo, tendo em vista suas necessidades e os meios existentes.

\subsubsection{Preco de um Servico Substituto}

Na composição da variāvel preço do serviço substituto, utilizamos dois serviços, o REPARTE e o Aluguel de Circuitos Telegráficos, que analisaremos a seguir.

O REPARTE consiste na utilização de um microcomputador de propriedade da EMBRATEL, exercendo as funções de uma central de co mutação, podendo interligar até 32 terminais ou 99 terminais no ca so de uma configuração expandida. o serviço permite a formação de redes fechadas de usuārios, com a possibilidade de interligação con a Rede Nacional de Telex. 
Em termos tarifārios, è cobrada uma tarifa fixa para cada teleimpressor conectado ao microcomputador, além de uma tarifa que remunere os circuitos interurbanos, nos casos em que um terminal e o microcomputador estiverem em diferentes āreas de tarifação.

Adicionalmente, é cobrada uma tarifa pelo número de entroncamentos com a rede pública, quando solicitados pelo usuário. Neste caso, as mensagens destinadas à rede püblica estão sujeitas às mes mas tarifas de utilização adotadas no serviço telex. o fluxo de mensagens entre terminais de uma mesma configuraçāo Reparte, nāo são tarifadas.

Outra caracteristica importante è o fato de a EMBRATEL ter alugado teleimpressores para usuārios deste serviço até março de 1986; o que certamente poderā ter influenciado as decisōes de alguns potenciais usuários do telex, dado o alto preço de mercado destes equipamentos.

Como não é possível quantificar as mensagens trafegadas entre os terminais de um mesmo usuário REPARTE, consideraremos, como variável de nosso modelo, a despesa mensal de um terminal Reparte e não o preço por mensagem, deste modo:

$\mathrm{DRP}=\mathrm{PAU}+\mathrm{DMC}+\mathrm{DMU}+\mathrm{DME}+\mathrm{PL}$

onde:

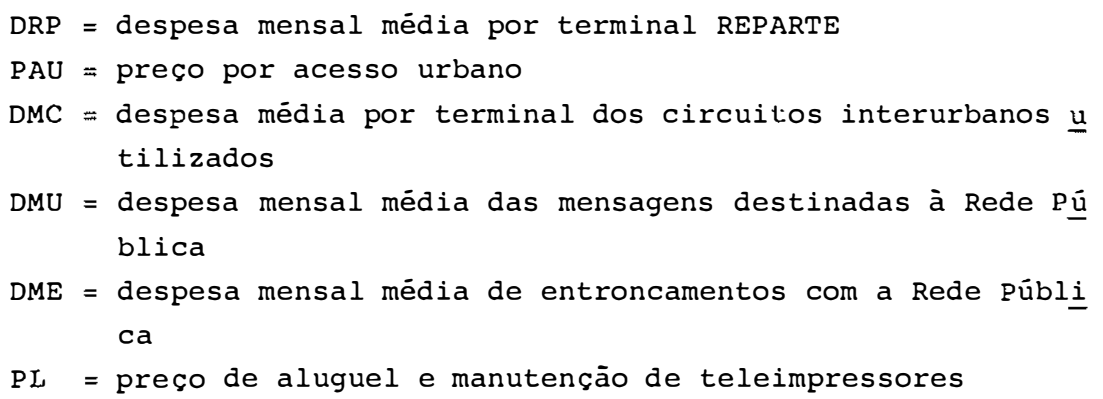

Supomos, tambēm, que todos os terminais sāo de propriedade da EMBRATEL e estão alugados aos usuários do serviço. 
Já o Serviço de Aluguel de Circuitos Telegráficos consiste ba sicamente em uma interligaçāo direta entre dois terminais, sem a intermediação de uma central de comutação. Tal serviço destina-sea usuários com perfil de alto tráfego entre pontos claramente determinados, habitualmente dois.

Neste serviço, é utilizado um sistema de tarifação no qual se cobra apenas um valor fixo pelo aluguel do circuito utilizado, variando em função da distância entre os terminais.

Assim como no caso anterior, a EMBRATEL também adotou o critê rio de alugar teleimpressores para os usuários deste serviço. Além disso, considerando que um mesmo circuito serve a dois terminais, a variável despesa média mensal por terminal será dada por:

$$
\mathrm{DCT}=\frac{\mathrm{PMCR}}{2}+\mathrm{PL}
$$

sendo:

$$
\begin{aligned}
\mathrm{DCT} & =\text { despesa média mensal por terminal } \\
\mathrm{PMCR} & =\text { preço médio por circuito } \\
\mathrm{PL} & =\text { preço de aluguel e manutenção de teleimpressores }
\end{aligned}
$$

\subsubsection{Nỉvel de Atividade Produtiva dos Usuários}

Para expressar a variāvel representativa do nível de atividade econômica dos usuários, foi utilizado o valor total dos empréstimos concedidos pelo setor bancário para os demais setores da eco nomia, uma vez que a maior parcela do tráfego total da rede pública tem sua origem no setor financeiro. Foi testado também a significância das variāveis ICM e IPI (Índice de produção Industrial), para expressar o nível de atividade econômica.

\subsubsection{Resultados Obtidos}

Apresentaremos, a seguir, os resultados obtidos pelo método dos mínimos quadrados ordinārios para um espaço amostral variando entre $A B R / 1980$ e MAR/1985. Todas as variāveis utilizadas foram transformadas para logaritmos. 
Tabe la

DEMANDA DESEJADA POR MENSAGENS

\begin{tabular}{|c|c|c|c|c|c|c|c|c|c|c|c|c|c|}
\hline \multirow{2}{*}{$\begin{array}{c}\text { VARIAVEL } \\
\text { DEPENDENTE }\end{array}$} & \multicolumn{9}{|c|}{ VARIAVEIS EXPLICATIVAS } & \multirow{2}{*}{$R^{2}$} & \multirow{2}{*}{ DW } & \multirow{2}{*}{$\begin{array}{c}F \\
(*)\end{array}$} & \multirow{2}{*}{$\begin{array}{c}\mathrm{R}^{2} \\
\text { AJUSTADO }\end{array}$} \\
\hline & CONSTANTE & $\begin{array}{l}\text { PRECO } \\
\text { TELEX }\end{array}$ & $\begin{array}{l}\text { EMPREST. } \\
\text { BANCOS }\end{array}$ & I C M & I P I & $\begin{array}{l}\text { TERMINAIS } \\
\text { ATIVADOS }\end{array}$ & $\begin{array}{l}\text { DESPESA } \\
\text { REPARTE }\end{array}$ & $\begin{array}{l}\text { DESPESA } \\
\text { ACT }\end{array}$ & \begin{tabular}{|c|} 
ONZE \\
DUREAYS
\end{tabular} & & & & \\
\hline PULSOS & $\begin{array}{r}-23,967 \\
-7,062\end{array}$ & $\begin{array}{l}-0,644 \\
-8,711\end{array}$ & $\begin{array}{l}0,961 \\
8,270\end{array}$ & - & $\ddot{-}$ & $\begin{array}{r}1,450 \\
22,663\end{array}$ & $\begin{array}{l}0,010 \\
2,961\end{array}$ & $\begin{array}{l}0,268 \\
3,927\end{array}$ & - & 0,985 & 1,588 & 710,9 & 0,984 \\
\hline PULSOS & $\begin{array}{l}2,398 \\
1,229\end{array}$ & $\begin{array}{l}-0,418 \\
-3,960\end{array}$ & - & $\begin{array}{l}-0,0001 \\
-0,004\end{array}$ & - & $\begin{array}{r}1,407 \\
13,878\end{array}$ & $\begin{array}{l}0,001 \\
0,244\end{array}$ & $\begin{array}{l}0,152 \\
1,452\end{array}$ & - & 0,966 & 1,116 & 307,5 & 0,965 \\
\hline PULSOS & $\begin{array}{r}-24,361 \\
-6,953\end{array}$ & $\begin{array}{l}-0,653 \\
-8,564\end{array}$ & $\begin{array}{l}0,965 \\
8,228\end{array}$ & $\begin{array}{l}0,006 \\
0,509\end{array}$ & - & $\begin{array}{r}1,461 \\
21,445\end{array}$ & $\begin{array}{l}0,011 \\
2,694\end{array}$ & $\begin{array}{l}0,278 \\
3,891\end{array}$ & - & 0,985 & 1,578 & 584,1 & 0,984 \\
\hline PULSOS & $\begin{array}{l}1,700 \\
0,869\end{array}$ & $\begin{array}{l}-0,400 \\
-3,800\end{array}$ & - & - & $\begin{array}{l}0,083 \\
0,781\end{array}$ & $\begin{array}{r}1,434 \\
14,134\end{array}$ & $\begin{array}{l}0,0013 \\
0,265\end{array}$ & $\begin{array}{l}0,160 \\
1,587\end{array}$ & - & 0,966 & 1,22 & 311,1 & 0,965 \\
\hline PULSOS & $\begin{array}{r}-24,714 \\
-7,199\end{array}$ & $\begin{array}{l}-0,626 \\
-8,327\end{array}$ & $\begin{array}{l}0,962 \\
8,315\end{array}$ & - & $\begin{array}{l}0,086 \\
1,225\end{array}$ & $\begin{array}{r}1,478 \\
21,838\end{array}$ & $\begin{array}{l}0,009 \\
2,913\end{array}$ & $\begin{array}{l}0,277 \\
4,048\end{array}$ & - & 0,985 & 1,746 & 597,9 & 0,984 \\
\hline PULSOS & $\begin{array}{r}-25,358 \\
-7,124\end{array}$ & $\begin{array}{l}-0,636 \\
-8,288\end{array}$ & $\begin{array}{l}0,967 \\
8,308\end{array}$ & $\begin{array}{l}0,095 \\
1,324\end{array}$ & $\begin{array}{l}0,008 \\
0,727\end{array}$ & $\begin{array}{r}1,497 \\
20,542\end{array}$ & $\begin{array}{l}0,011 \\
2,786\end{array}$ & $\begin{array}{l}0,293 \\
4,068\end{array}$ & - & 0,986 & 1,745 & 508,1 & 0,984 \\
\hline PULSOS & $\begin{array}{r}-20,916 \\
-5,946\end{array}$ & $\begin{array}{l}-0,541 \\
-6,311\end{array}$ & $\begin{array}{l}0,891 \\
7,445\end{array}$ & - & - & $\begin{array}{r}1,394 \\
21,706\end{array}$ & $\begin{array}{l}0,011 \\
3,223\end{array}$ & $\begin{array}{l}0,197 \\
2,795\end{array}$ & $\begin{array}{l}\mathrm{S} \\
\mathrm{S}\end{array}$ & 0,98 & 1,536 & 250,8 & 0,973 \\
\hline
\end{tabular}

(*) para k-1 e n-k graus de liberdade, sendo $n$ igual a 60 e $k$ igual ao número de parāmetros estimados. 
Na tabela anterior, destaca-se que a inclusāo das onze dummys nāo provocou efeitos significativos nos resultados da equação; ten do a variāvel empréstimos bancārios demonstrado ser um bom indicador do nível de atividade produtiva dos usuários. Consequentemente selecionamos a primeira equaçāo como aquela que apresentou os melhores resultados tanto quanto aos valores das estatisticas $t$ de Student, quanto ao valor apresentado pelo coeficiente de determina ção e pelo teste de Durbin Watson. Adicionalmente, os sinais dos coeficientes estimados por esta equação, são os previstos pela Teo ria Econômica.

Ainda dentro do item de Demanda por Mensagem, realizaremos al guns testes com a equação de pulsos por terminal. Para tanto, utilizamos as mesmas variāveis explicativas testadas na equação anterior.

Nesta equaçāo, a variāvel terminais ativados assume um papel que merece ser comentado. No caso anterior, esta variāvel represen tava uma restrição quanto ao potencial māximo de geração de tráfego na rede pública. Nesta, cada novo terminal ativado na rede representa uma ampliação das possibilidades de trāfego entre os demais. Melhor explicando: suponhamos um caso hipotético de uma rede composta por apenas dois terminais, A e B. Nesta situação, o fluxo de mensagens na rede segue apenas dois caminhos, ou seja, de $A$ em direção a $B$ e de $B$ em direção a $A$. Com a ativação de mais um termi nal, digamos C, o terminal A poderá enviar mensagens para B e $C$, e assim sucessivamente, para cada novo terminal ativado, as possibilidades de tráfego para um já existente se elevam. Deste modo, espera-se que o tráfego médio aumente à medida que a rede cresce.

o quadro seguinte apresenta os resultados obtidos nos testes realizados com esta equação. 
Tabela 2

DEMANDA DE PULSOS POR TERMINAL

\begin{tabular}{|c|c|c|c|c|c|c|c|c|c|c|c|c|c|}
\hline \multirow{2}{*}{$\begin{array}{l}\text { VARI ANVEL } \\
\text { DEPENDENTE }\end{array}$} & \multicolumn{9}{|c|}{ VARIAVEIS EXPLICATIVAS } & \multirow{2}{*}{$\mathrm{R}^{2}$} & \multirow[b]{2}{*}{ DW } & \multirow{2}{*}{$\underset{(\star)}{F}$} & \multirow{2}{*}{$\begin{array}{c}\mathrm{R}^{2} \\
\text { AJUS TAD }\end{array}$} \\
\hline & CONSTANTE & $\begin{array}{l}\text { PRECO } \\
\text { TELEX }\end{array}$ & $\begin{array}{c}\text { EMPRES T. } \\
\text { BANCOS }\end{array}$ & I C M & I P I & $\begin{array}{c}\text { TERMINAIS } \\
\text { ATIVADOS }\end{array}$ & \begin{tabular}{l|} 
DESPESA \\
REPAR TE
\end{tabular} & $\begin{array}{c}\text { DESPESA } \\
\text { ACT }\end{array}$ & $\begin{array}{c}\text { ONZE } \\
\text { DUMMYS }\end{array}$ & & & & \\
\hline $\begin{array}{l}\text { PULSOS } \\
\text { POR } \\
\text { TERHINAL }\end{array}$ & $\begin{array}{r}-23,991 \\
-7,082\end{array}$ & $\begin{array}{l}-0,644 \\
-8,727\end{array}$ & $\begin{array}{l}0,962 \\
8,291\end{array}$ & - & $\begin{array}{l}- \\
-\end{array}$ & $\begin{array}{l}0,450 \\
7,046\end{array}$ & $\begin{array}{l}0,010 \\
2,985\end{array}$ & $\begin{array}{l}0,269 \\
3,944\end{array}$ & $\begin{array}{l}- \\
-\end{array}$ & 0,886 & 1,583 & 83,9 & 0,884 \\
\hline $\begin{array}{l}\text { PULSOS } \\
\text { POR } \\
\text { TERMINAL }\end{array}$ & $\begin{array}{r}-20,923 \\
-5,962\end{array}$ & $\begin{array}{l}-0,541 \\
-6,318\end{array}$ & $\begin{array}{l}0,892 \\
7,463\end{array}$ & - & - & $\begin{array}{l}0,394 \\
6,151\end{array}$ & $\begin{array}{l}0,011 \\
3,249\end{array}$ & $\begin{array}{l}0,198 \\
2,813\end{array}$ & s & 0,919 & 1,513 & 30,6 & 0,888 \\
\hline $\begin{array}{l}\text { PULSOS } \\
\text { POR } \\
\text { TERMINAL }\end{array}$ & $\begin{array}{r}-22,107 \\
-6,158\end{array}$ & $\begin{array}{l}-0,537 \\
-6,338\end{array}$ & $\begin{array}{l}0,898 \\
7,579\end{array}$ & - & $\begin{array}{l}0,135 \\
1,334\end{array}$ & $\begin{array}{l}0,432 \\
6,205\end{array}$ & $\begin{array}{l}0,010 \\
2,696\end{array}$ & $\begin{array}{l}0,198 \\
2,843\end{array}$ & s & 0,923 & 1,614 & 29,5 & 0,892 \\
\hline $\begin{array}{l}\text { PULSOS } \\
\text { POR } \\
\text { TERMINAZ }\end{array}$ & $\begin{array}{r}-22,346 \\
-5,675\end{array}$ & $\begin{array}{l}-0,543 \\
-5,897\end{array}$ & $\begin{array}{l}0,901 \\
7,426\end{array}$ & $\begin{array}{l}0,002 \\
0,157\end{array}$ & $\begin{array}{l}0,142 \\
1,279\end{array}$ & $\begin{array}{l}0,438 \\
5,433\end{array}$ & $\begin{array}{l}0,010 \\
2,435\end{array}$ & $\begin{array}{l}0,202 \\
2,717\end{array}$ & $\begin{array}{l}\mathrm{S} \\
\mathrm{S}\end{array}$ & 0,923 & 1,602 & 27,2 & 0,889 \\
\hline $\begin{array}{l}\text { PULSOS } \\
\text { POR } \\
\text { TERMINAZ }\end{array}$ & $\begin{array}{r}-20,542 \\
-5,546\end{array}$ & $\begin{array}{l}-0,529 \\
-5,744\end{array}$ & $\begin{array}{l}0,886 \\
7,281\end{array}$ & $\begin{array}{l}-0,005 \\
-0,355\end{array}$ & - & $\begin{array}{l}0,385 \\
5,537\end{array}$ & $\begin{array}{l}0,011 \\
2,527\end{array}$ & $\begin{array}{l}0,191 \\
2,561\end{array}$ & $\begin{array}{l}\mathrm{S} \\
\mathrm{S}\end{array}$ & 0,920 & 1,565 & 28,3 & 0,887 \\
\hline $\begin{array}{l}\text { PULSOS } \\
\text { POR } \\
\text { TERMINAL }\end{array}$ & $\begin{array}{r}-24,735 \\
-7,217\end{array}$ & $\begin{array}{l}-0,626 \\
-8,344\end{array}$ & $\begin{array}{l}0,962 \\
8,336\end{array}$ & - & $\begin{array}{l}0,086 \\
1,221\end{array}$ & $\begin{array}{l}0,478 \\
7,073\end{array}$ & $\begin{array}{l}0,009 \\
2,937\end{array}$ & $\begin{array}{l}0,278 \\
4,046\end{array}$ & $\begin{array}{l}\mathrm{s} \\
\mathrm{s}\end{array}$ & 0,889 & 1,740 & 70,9 & 0,844 \\
\hline
\end{tabular}

(*) para k-l e n-k graus de liberdade, sendo $n$ igual a 60 e k igual ao nümero de parāmetros estimados, 
Comparando-se os resultados apresentados nas duas tabelas anteriores, pode-se observar que a influência das sazonalidades se mostrou mais forte na equação de pulsos por terminal do que na equação de pulsos totais. Isto pode ser explicado por ser a equação de pulsos totais influenciada pelo ingresso de tráfego decorrente das ativaçōes mensais de novos terminais, o que vem a atenuar o efeito de oscilaçōes causadas por sazonalidades.

Selecionamos a segunda equação da tabela anterior, como aquela que melhor expressa a equação de pulsos por terminal. Tal equacão difere daquela selecionada para expressar o total de pulsos, a penas no que tange à inclusão de onze dummys.

\section{2 A Demanda Desejada por Terminais}

Conforme analisamos anteriormente, a demanda desejada por ter minais é proporcional à demanda desejada por mensagens; portanto, as mesmas variāveis que influenciam a segunda deverão influenciar a primeira. Temos, então, que:

$$
K D=f(P m, P s, Q)
$$

onde:

$$
\begin{aligned}
& \mathrm{KD}=\text { demanda desejada por terminais } \\
& \mathrm{Pm}=\text { preço médio da mensagem telex } \\
& \mathrm{Ps}=\text { preço de serviços substitutos } \\
& \mathrm{Q}=\text { nível de atividade produtiva dos usuários }
\end{aligned}
$$

Nestes testes, utilizamos os mesmós serviços substitutos descritos anteriormente, enquanto que a variável representativa do ní vel de atividade produtiva merece maiores comentārios.

Apesar de ser o setor financeiro o responsável pela maior par cela na geração de tráfego na rede, o mesmo não ocorre quanto ao número de terminais, uma vez que é o setor industrial o responsável pela maior participação no total dos terminais ativados. o que nos 
leva a esperar que a variāvel IPI, indice de produção industrial, seja mais apropriada para expressar o nivel de atividade econômica dos usuários.

Alēm das variāveis jā analisadas, deverá ser considerada tambēm na equação, o número de localidades interligadas pela Rede Nacional de Telex, pois quando uma nova Localidade passa a ser atendi da, todos os potenciais usuários nela "residentes" são induzidos a se inscreverem no serviço.

o quadro seguinte apresenta os resultados obtidos para esta equação pelo método dos mínimos quadrados ordinārios, adotando-se como espaço amostral o período entre abril de 1980 e março de 1985. Foram, também, realizadas transformaçōes logaritmicas em todas as variāveis utilizadas.

Como em todas as equaçōes da tabela anterior, o teste de Durbin-Watson apresentou valores relativamente baixos, indicando a existência de autocorrelação dos resíduos. Realizamos novos testes incluindo a variável dependente defasada no conjunto das variáveis explicativas. 
Tabela 3

DEMANDA DESEJADA POR TERMINAIS

\begin{tabular}{|c|c|c|c|c|c|c|c|c|c|c|c|c|c|c|}
\hline \multirow{2}{*}{$\begin{array}{l}\text { VARIÁVEL } \\
\text { JEPENDENTE }\end{array}$} & \multicolumn{10}{|c|}{ VARIAVEIS EXPLICATIVAS } & \multirow[b]{2}{*}{$\mathrm{R}^{2}$} & \multirow[b]{2}{*}{ DW } & \multirow[b]{2}{*}{$\underset{(*)}{F}$} & \multirow[b]{2}{*}{$\begin{array}{c}\mathbf{R}^{\mathbf{2}} \\
\text { AJ USTADO }\end{array}$} \\
\hline & CONSTANTE & $\begin{array}{l}\text { PRECCO } \\
\text { TELEX }\end{array}$ & $\begin{array}{l}\text { EMPREST. } \\
\text { BANCOS }\end{array}$ & I C $\mathrm{M}$ & $\begin{array}{lll}\text { I } & \text { P I } & \text { I }\end{array}$ & $\begin{array}{l}\text { DESPESA } \\
\text { REPARTE }\end{array}$ & $\begin{array}{c}\text { DESPESA } \\
\text { ACT }\end{array}$ & $\begin{array}{c}\text { LOCALIDADE } \\
\text { ATEND.IDAS }\end{array}$ & TERMINAIS & \begin{tabular}{|c|} 
ONZE \\
DUMMYS
\end{tabular} & & & & \\
\hline INSCRI ÇŌES & $\begin{array}{r}13,815 \\
9,013\end{array}$ & $\begin{array}{l}-0,091 \\
-2,507\end{array}$ & $\begin{array}{r}-0,242 \\
4,417\end{array}$ & - & - & $\begin{array}{l}0,0005 \\
0,301\end{array}$ & $\begin{array}{l}0,162 \\
4,969\end{array}$ & $\begin{array}{r}0,314 \\
22,374\end{array}$ & & $\overline{-}$ & 0,977 & 0,718 & 458,8 & 0,976 \\
\hline INSCRICŌES & $\begin{array}{r}7,661 \\
18,231\end{array}$ & $\begin{array}{l}-0,135 \\
-3,556\end{array}$ & $\overline{-}$ & $\begin{array}{l}-0,015 \\
-2,421\end{array}$ & - & $\left|\begin{array}{l}-0,0005 \\
-0,239\end{array}\right|$ & $\begin{array}{l}0,176 \\
4,882\end{array}$ & $\begin{array}{r}0,310 \\
19,415\end{array}$ & & $\overline{-}$ & 0,972 & 0,830 & 370,1 & 0,971 \\
\hline INSCRI COES & $\begin{array}{r}6,957 \\
15,161\end{array}$ & $\begin{array}{l}-0,143 \\
-3,615\end{array}$ & - & - & $\begin{array}{l}0,042 \\
1,085\end{array}$ & $\begin{array}{l}0,003 \\
1,550\end{array}$ & $\begin{array}{l}0,199 \\
5,377\end{array}$ & $\begin{array}{r}0,327 \\
18,993\end{array}$ & & $\overline{-}$ & 0,969 & 0,799 & 340,3 & 0,968 \\
\hline INSCRI Ç̃ES & $\begin{array}{r}14,375 \\
9,834\end{array}$ & $\begin{array}{l}-0,067 \\
-1,950\end{array}$ & $\begin{array}{l}-0,251 \\
-4,932\end{array}$ & $\begin{array}{l}-0,016 \\
-3,041\end{array}$ & $\begin{array}{l}0,026 \\
0,831\end{array}$ & $\begin{array}{l}-0,003 \\
-1,637\end{array}$ & $\begin{array}{l}0,145 \\
4,618\end{array}$ & $\begin{array}{r}0,307 \\
21,190\end{array}$ & & - & 0,981 & 0,838 & 381,6 & 0,980 \\
\hline INSCRI ÇŌES & $\begin{array}{l}14,866 \\
28,059\end{array}$ & $\begin{array}{l}0,231 \\
2,441\end{array}$ & - & - & $\begin{array}{l}-0,215 \\
-2,169\end{array}$ & $\begin{array}{l}0,013 \\
2,961\end{array}$ & $\begin{array}{l}-0,359 \\
-5,829\end{array}$ & $\overline{-}$ & & - & 0,764 & 0,334 & 358,6 & 0,759 \\
\hline INSCRIÇẼ & $\begin{array}{r}7,214 \\
18,315\end{array}$ & $\begin{array}{l}-0,151 \\
-3,864\end{array}$ & - & - & - & $\begin{array}{l}0,003 \\
1,566\end{array}$ & $\begin{array}{l}0,194 \\
5,279\end{array}$ & $\begin{array}{r}0,320 \\
19,844\end{array}$ & & - & 0,969 & 0,831 & 423,7 & 0,968 \\
\hline INSCRI COES & $\begin{array}{r}24,429 \\
5,435\end{array}$ & $\begin{array}{l}0,307 \\
3,126\end{array}$ & $\begin{array}{l}-0,359 \\
-2,142\end{array}$ & - & $\begin{array}{l}-0,211 \\
-2,192\end{array}$ & $\begin{array}{l}0,009 \\
2,071\end{array}$ & $\begin{array}{l}-0,390 \\
-6,351\end{array}$ & - & & - & 0,782 & 0,455 & 386,2 & 0,778 \\
\hline INSCRI C̋̃ES & $\begin{array}{l}2,1868 \\
3,3632\end{array}$ & $\begin{array}{l}-0,0949 \\
-2,4719\end{array}$ & - & $\overline{-}$ & - & $\begin{array}{l}-0,0012 \\
-0,6682\end{array}$ & $\begin{array}{l}0,1852 \\
4,949\end{array}$ & - & $\begin{array}{r}0,6875 \\
19,2977\end{array}$ & - & 0,967 & 0,659 & 402,8 & 0,966 \\
\hline INSCRI CÕES & $\begin{array}{l}4,9577 \\
3,9502\end{array}$ & $\begin{array}{l}-0,1323 \\
-3,351\end{array}$ & - & $\overline{-}$ & - & $\begin{array}{l}0,0008 \\
0,4437\end{array}$ & $\begin{array}{l}0,1973 \\
5,4.78\end{array}$ & $\begin{array}{l}0,1854 \\
2,539\end{array}$ & $\begin{array}{l}0,2973 \\
1,889\end{array}$ & - & 0,971 & 0,819 & 355,5 & 0,970 \\
\hline INSCR I ÇŌES & $\begin{array}{c}6,8905 \\
15,763\end{array}$ & $\begin{array}{l}-0,1909 \\
-3,9318\end{array}$ & - & - & - & $\begin{array}{l}0,0017 \\
0,8659\end{array}$ & $\begin{array}{l}0,2229 \\
5,4423\end{array}$ & $\begin{array}{r}0,3301 \\
18,7297\end{array}$ & $\overline{-}$ & $\begin{array}{l}\mathrm{S} \\
\mathrm{S}\end{array}$ & 0,974 & 0,855 & 107,8 & 0,964 \\
\hline
\end{tabular}


Tabela 4

\begin{tabular}{|c|c|c|c|c|c|c|c|c|c|c|c|c|c|c|c|}
\hline \multirow{2}{*}{$\begin{array}{c}\text { VARIKVEL } \\
\text { DEPENDENTE }\end{array}$} & \multicolumn{10}{|c|}{ VARIÁVEIS EXPLICATIVAS } & \multirow[b]{2}{*}{$R^{2}$} & \multirow[b]{2}{*}{ DW } & \multirow[b]{2}{*}{$\mathrm{H}$} & \multirow[b]{2}{*}{$\begin{array}{c}F \\
(*)\end{array}$} & \multirow[b]{2}{*}{$\begin{array}{c}\mathrm{R}^{2} \\
\text { AJUSTADO }\end{array}$} \\
\hline & CONSTANTE & $\begin{array}{l}\text { PRECO } \\
\text { TELEX }\end{array}$ & \begin{tabular}{|c|} 
EMPREST. \\
BANCOS
\end{tabular} & I C M & $\begin{array}{lll}\text { I } & \text { P I } & \\
\end{array}$ & $\begin{array}{l}\text { DESPESA } \\
\text { REPARTE }\end{array}$ & \begin{tabular}{|c} 
DESPESA \\
ACT
\end{tabular} & $\begin{array}{r}\text { LOCALID ADE: } \\
\text { ATEND IDAS }\end{array}$ & $\begin{array}{l}\text { INSC }_{(t-1)} \\
\end{array}$ & \begin{tabular}{|c|} 
ON2E \\
DUMBNYS
\end{tabular} & & & & & \\
\hline INSCRICOES & $\begin{array}{r}7,214 \\
18,315\end{array}$ & $\begin{array}{l}-0,151 \\
-3,864\end{array}$ & - & - & - & $\begin{array}{l}0,003 \\
1,566\end{array}$ & $\begin{array}{l}0,194 \\
5,279\end{array}$ & $\begin{array}{r}0,320 \\
19,844\end{array}$ & - & - & 0,969 & 0,831 & - & 423,7 & 0,969 \\
\hline INSCRICOES & $\begin{array}{l}0,4062 \\
1,4947\end{array}$ & $\begin{array}{l}-0,036 \\
-3,274\end{array}$ & - & - & - & $\begin{array}{l}0,0009 \\
1,977\end{array}$ & $\begin{array}{l}0,026 \\
2,270\end{array}$ & $\begin{array}{l}0,0123 \\
1,0135\end{array}$ & $\begin{array}{c}0,9339 \\
27,117\end{array}$ & - & 0,998 & 2,073 & $-0,297$ & 5012,0 & 0,997 \\
\hline INSCRI COES & $\begin{array}{l}0,2014 \\
1,107\end{array}$ & $\begin{array}{l}-0,030 \\
-3,255\end{array}$ & - & - & - & $\begin{array}{l}0,0009 \\
1,9604\end{array}$ & $\begin{array}{l}0,0175 \\
2,2679\end{array}$ & - & $\begin{array}{c}0,9665 \\
79,884\end{array}$ & - & 0,998 & 2,002 & $-0,009$ & 6260,6 & 0,997 \\
\hline INSCRICOES & $\begin{array}{l}0,223 \\
0,406\end{array}$ & $\begin{array}{l}-0,030 \\
-2,839\end{array}$ & $\begin{array}{l}-0,0007 \\
-0,0417\end{array}$ & - & - & $\begin{array}{l}0,0009 \\
1,8612\end{array}$ & $\begin{array}{l}0,017 \\
2,084\end{array}$ & - & $\begin{array}{r}0,966 \\
76,098\end{array}$ & - & 0,998 & $2,000 z$ & -00013 & 4918,6 & 0,997 \\
\hline INSCRICOES & $\begin{array}{c}0,312 \\
: 1,5517\end{array}$ & $\begin{array}{l}-0,028 \\
-2,994\end{array}$ & - & $\begin{array}{l}-0,0022 \\
-1,258\end{array}$ & - & $\begin{array}{l}0,0004 \\
0,756\end{array}$ & $\begin{array}{l}0,0152 \\
1,9289\end{array}$ & - & $\begin{array}{r}0,9613 \\
75,4119\end{array}$ & - & 0,998 & 2,016 & $-0,0636$ & 5061,9 & 0,997 \\
\hline INSCRI Ø̄ES & $\begin{array}{l}0,2168 \\
0,7248\end{array}$ & $\begin{array}{l}-0,0306 \\
-2,117\end{array}$ & - & - & - & $\begin{array}{l}0,0008 \\
1,4301\end{array}$ & $\begin{array}{l}0,0133 \\
0,9578\end{array}$ & $\begin{array}{l}-0,001 \\
-0,0692\end{array}$ & $\begin{array}{r}0,9683 \\
24,1881\end{array}$ & $\begin{array}{l}\mathrm{S} \\
\mathrm{S}\end{array}$ & 0,998 & 1,922 & 0,33 & 1479,3 & 0,997 \\
\hline INSCRICÕES & $\begin{array}{l}0,3895 \\
1,4156\end{array}$ & $\begin{array}{l}-0,0358 \\
-3,1651\end{array}$ & - & - & $\begin{array}{l}0,0057 \\
0,553\end{array}$ & $\begin{array}{l}0,0009 \\
1,9661\end{array}$ & $\begin{array}{l}0,0273 \\
2,3163\end{array}$ & $\begin{array}{l}0,014 \\
1,1128\end{array}$ & $\begin{array}{c}0,9313 \\
26,637\end{array}$ & $\begin{array}{l}- \\
-\end{array}$ & 0,998 & 2,0891 & $-0,32$ & 4122,9 & 0,997 \\
\hline İASCR I COES & $\begin{array}{l}0,2326 \\
1,207\end{array}$ & $\begin{array}{l}-0,031 \\
-2,733\end{array}$ & - & - & - & $\begin{array}{l}0,0008 \\
1,4448\end{array}$ & $\begin{array}{l}0,014 \\
1,698\end{array}$ & - & $\begin{array}{l}0,9657 \\
75,628\end{array}$ & $\begin{array}{l}\mathrm{S} \\
\mathrm{S}\end{array}$ & 0,998 & 1,932 & 0,278 & 1613,7 & 0,997 \\
\hline
\end{tabular}

(*) para k-1 e n-k graus de liberdade, sendo n igual a 60 e k igual ao nümero de parâmetros estimados. 
Nesta tabela, pode ser observado que a inclusāo da defasagem da variāvel dependente, tornou nāo significativa a variāvel número de localidades atendidas. Todavia, os resultados da estatistica de Durbin-Watson e do teste $\mathrm{H}$, indicam que o problema de autocorrelaçāo dos residuos foi resolvido.

A obtençāo de estimativas do coeficiente da variāvel endógena defasada, com valores próximos de um, nas diversas equaçōes testadas, indica que a demanda desejada por terminais responde lentamen te a alteraçōes nas demais variāveis; ou seja, a ocorrência de uma alteraçāo, por exemplo, no valor das tarifas em um determinado mês, produziria efeitos muito fracos no próprio mês, tornando-se mais $\underline{e}$ fetivas tais influências nos meses seguintes.

Os coeficientes das variāveis ICM e emprēstimos bancārios, as sumiram sinais contrārios aos esperados, além do que, a estatística t da variável IPI, apresentou valores relativamente baixos, em alguns casos, enquanto que, em outros, os sinais dos coeficientes foram também negativos.

Nas equaçōes onde estas três variáveis foram omitidas, o coeficiente de determinaçāo, bem como, a estatistica de Durbin-Watson e o teste $\mathrm{H}$ nāo foram comprometidos, desde que incluída a variāvel defasada das inscriçōes no conjunto das variāveis explicativas. Por isto selecionamos a terceira equaçāo como aquela que apre sentou os melhores resultados.

3. 3 Estoque de Terminais Ativados na Rede

Considerando a hipótese apresentada anteriormente de que a vạ riaçāo física do número de terminais ativados em um determinado mês è uma funçāo das variaçōes líquidas das inscriçōes, ocorridas no próprio mês e em meses anteriores:

$$
K E_{t}-K E_{t-1}=E\left(K D_{t}-K D_{t-1}, \cdots, K D_{t-n}-K D_{t-n-1}\right)
$$


onde:

$$
\begin{aligned}
& \mathrm{KE}=\text { estoque de terminais ativados } \\
& \mathrm{KD}=\text { demanda desejada de terminais }
\end{aligned}
$$

Para a obtençāo dos coeficientes desta equação, adotamos o método para tratamento de defasagens desenvolvido por Almon. Além disso, tornou-se necessārio que utilizássemos o artifício de elimi nar o termo constante, permitindo assim que o somatório dos coefí cientes obtidos se igualassem à unidade. Utilizamos a seguinte equação:

$$
K E_{t}-K E_{t-1}=a_{1} z_{1 t}+a_{2} z_{2 t}+a_{3} z_{3 t}+a_{4} z_{4 t}+a_{5} z_{5 t}+e_{t}
$$

onde: $z_{n t}=\sum_{i=0}^{k} i^{n-1} x_{t-i}$, sendo $n=1,2,3,4,5$

$$
x_{t-1}=\left(K D_{t-i}-K D_{t-i-1}\right)
$$

Sendo os coeficientes da equaçāo original obtidos pela seguin te fórmula:

$$
b_{i}=a_{1}+a_{2} i^{1}+a_{3} i^{2}+a_{4} i^{3}+a_{5} i^{4}
$$

A tabela seguinte apresenta os resultados obtidos nos testes realizados para um período entre Abril de 1980 e Março de 1985, adicionados dos respectivos "lags" utilizados. 
Tabela 5

FLUXO DAS ATIVACOES DE TERMINAIS

\begin{tabular}{|c|c|c|c|c|c|c|c|c|c|c|c|c|}
\hline \multirow[b]{2}{*}{$\begin{array}{c}\text { VARIÃVEL } \\
\text { I NDEPENDENTE }\end{array}$} & \multicolumn{7}{|c|}{ VARIAVEIS EXPLICATIVAS } & \multirow{2}{*}{$\begin{array}{c}\text { NUEERO } \\
\text { DE } \\
\text { LAGS }\end{array}$} & \multirow{2}{*}{$\begin{array}{c}\text { SOMA } \\
\text { DOS } \\
\text { COEFIC. }\end{array}$} & \multirow[b]{2}{*}{$R^{2}$} & \multirow[b]{2}{*}{ D.W } & \multirow[b]{2}{*}{$\begin{array}{c}\mathbf{R}^{\mathbf{2}} \\
\text { A.JUSTADO }\end{array}$} \\
\hline & CONSTANTE & $z_{1}$ & $z_{2}$ & $z_{3}$ & $2_{4}$ & $z_{5}$ & $\begin{array}{c}\text { ONZE } \\
\text { DUMNYS }\end{array}$ & & & & & \\
\hline$K E_{t}-K E_{t-1}$ & - & $\begin{array}{l}0,432 \\
4,492\end{array}$ & $\begin{array}{l}-3,787 \\
-2,92\end{array}$ & $\begin{array}{r}10,56 \\
2,12\end{array}$ & $\begin{array}{r}-11,75 \\
1,61\end{array}$ & $\begin{array}{l}4,66 \\
1,30\end{array}$ & - & 24 & 1,029 & 0,865 & 1,15 & 0,855 \\
\hline$K E_{t}-K E_{t-1}$ & - & $\begin{array}{l}0,374 \\
4,732\end{array}$ & $\begin{array}{l}-3,765 \\
-3,343\end{array}$ & $\begin{array}{c}12,03 \\
2,702\end{array}$ & $\begin{array}{r}-15,178 \\
-2,309\end{array}$ & $\begin{array}{l}6,66 \\
2,06\end{array}$ & - & 30 & 1,053 & 0,885 & 1,24 & 0,877 \\
\hline$K E_{t}-K E_{t-1}$ & - & $\begin{array}{l}0,279 \\
5,116\end{array}$ & $\begin{array}{l}-3,038 \\
-3,994\end{array}$ & $\begin{array}{r}10,652 \\
3,578\end{array}$ & $\begin{array}{r}-14,612 \\
-3,336\end{array}$ & $\begin{array}{l}6,883 \\
3,192\end{array}$ & - & 36 & 1,05 & 0,905 & 1,507 & 0,898 \\
\hline$K E_{t}-K E_{t-1}$ & - & $\begin{array}{l}0,201 \\
3,418\end{array}$ & $\begin{array}{l}-2,285 \\
-2,833\end{array}$ & $\begin{array}{l}8,243 \\
2,594\end{array}$ & $\begin{array}{r}-11,54 \\
-2,44\end{array}$ & $\begin{array}{l}5,507 \\
2,342\end{array}$ & $\begin{array}{l}\mathrm{S} \\
\mathrm{S}\end{array}$ & 36 & 0,649 & 0,936 & 1,758 & 0,914 \\
\hline
\end{tabular}


Observa-se, na tabela anterior, que a introdução de onze dummys impede que a soma dos coeficientes da equação original seja igual à unidade. Por outro lado, a equação que utiliza 36 lagas pa ra um polinômio do quarto grau, foi a que apresentou os melhores resultados.

Nāo nos foi possível realizar testes com polinômios de grau superior a quatro, tendo em vista um mal condicionamento das matri zes no processo de cālculo.

\section{4 Teste de Modelo Recursivo}

Conforme analisado anteriormente, as equaçōes que compōem nos so modelo formam um sistema recursivo, uma vez que o curso das influências entre as equaçōes è unidirecional. Tal fato nos permite utilizar o Método dos Mínimos Quadrados ordinārios para todasas equaçōes, uma vez que os termos de perturbação destas, são "não correlacionados" para o mesmo período.

Com base nas equaçōes selecionadas nas tabelas anteriores, rea lizamos alọmas simulaçōes com o modelo como um todo. Sendo assim, os valores estimados das variāveis dependentes, foram comparados com os respectivos valores observados no período analisado. Neste item apresentaremos o quadro resumo das estatisticas referentes a cada equação, que estão expressas nas tabelas a seguir.

Tabe 1 a 6

RESUMO DAS ESTATISTICAS

EQUAČ̃O DE PULSOS TOTAIS

\begin{tabular}{lcccc}
\hline & $\begin{array}{l}\text { VALOR }(*) \\
\text { OBSERVADO }\end{array}$ & $\begin{array}{l}\text { VALOR }(*) \\
\text { ESTIMADO }\end{array}$ & $\begin{array}{c}\text { ERRO } \\
\text { ESTIMAÇO }\end{array}$ & $\begin{array}{c}\text { ERRO } \\
\text { PERCENTAL }\end{array}$ \\
\cline { 2 - 2 } & 19,319 & 19,328 & 0,0089 & 0,047 \\
$\begin{array}{l}\text { Desvio } \\
\text { Padráo }\end{array}$ & 0,327 & 0,326 & 0,038 & 0,197 \\
\hline
\end{tabular}

(*) Valores em Logaritmos. 


\author{
Tabela 7 \\ RESUMO DAS ESTATISTICAS \\ EQUACAO DE PULSOS POR TERMINAL
}

\begin{tabular}{lcccc}
\hline & $\begin{array}{l}\text { VALOR (*) } \\
\text { OBSERVACAO }\end{array}$ & $\begin{array}{c}\text { VALOR }(*) \\
\text { ESTIMADO }\end{array}$ & $\begin{array}{c}\text { ERRO DE } \\
\text { ESTIMACAO }\end{array}$ & $\begin{array}{c}\text { ERRO } \\
\text { PERCENTUAL }\end{array}$ \\
\hline $\begin{array}{l}\text { Média } \\
\text { Desvio }\end{array}$ & 8,556 & 8,562 & 0,005 & 0,063 \\
Padräo & 0,119 & 0,119 & 0,032 & 0,375 \\
\hline
\end{tabular}

(*) Valores em Logaritmos.

Tabela 8

RESUMO DAS ESTATISTICAS

EQUACAO DE DEMANDA DESEJADA POR TERMINAIS

\begin{tabular}{lcccc}
\hline & $\begin{array}{l}\text { VALOR (*) } \\
\text { OBSERVADO }\end{array}$ & $\begin{array}{c}\text { VALOR }(*) \\
\text { ESTIMADO }\end{array}$ & $\begin{array}{c}\text { ERRO DE } \\
\text { ESTIMACÃO }\end{array}$ & $\begin{array}{c}\text { ERRO } \\
\text { RERCENTUAL }\end{array}$ \\
\hline $\begin{array}{l}\text { Média } \\
\text { Desvio }\end{array}$ & 11,028 & 11,028 & 0,0004 & 0,004 \\
Padräo & 0,128 & 0,127 & 0,013 & 0,118 \\
\hline
\end{tabular}

(*) Valor em Logaritmos.

Tabela 9

RESUMO DAS ESTATISTICAS

EQUAÇAO DO FLUXO DE ATIVAÇOES DE TERMINAIS

\begin{tabular}{lcccc}
\hline & $\begin{array}{c}\text { VALOR } \\
\text { OBSERVADO }\end{array}$ & $\begin{array}{c}\text { VALOR } \\
\text { ESTIMADO }\end{array}$ & $\begin{array}{c}\text { ERRO DE } \\
\text { ESTIMAÇA }\end{array}$ & $\begin{array}{c}\text { ERRO } \\
\text { PERCENTUAL }\end{array}$ \\
\hline Média & $48.252,2$ & $48.356,2$ & 104,3 & 0,308 \\
$\begin{array}{l}\text { Desvió } \\
\text { Padräo }\end{array}$ & $10.432,7$ & $10.165,7$ & 898,4 & 1,6111 \\
\hline
\end{tabular}


Podemos observar que os resultados expressos nos quadros ante riores indicam que o erro percentual médio obtido foi inferior a 1 \& nas quatro equações do modelo.

\section{CONSIDERACOES FINAIS}

Considerando os resultados apresentados nos itens anteriores, podemos concluir que os usuários do serviço de telex nacional, quan do tomados em termos agregados, sāo muito pouco sensíveis a variações nos preços e tarifas relativas ao serviço. Isto deve-se ao fa to de que a elasticidade estimada para a equaçāo do tráfego total de mensagens, com relaçāo ao preço do serviço é de $-0,64$, enquanto que a elasticidade estimada para a equaçāo do trāfego por terminal, com relaçāo à mesma variāvel, é de $-0,54$. Outro fato importante re gistrado na equaçāo de pulsos por terminal é que a obtençāo de um coeficiente com sinal positivo para a variável nümero de terminais indica que o tráfego médio por terminal aumenta à medida que a rede cresce.

De uma maneira geral, pode-se concluir que todas as mudanças na mecãnica tarifäria, que por ventura venham a provocar aumentos no valor real cobrado pelo serviço, resultariam em variaçōes positivas nos níveis de receita, uma vez que a reduçāo no trāfego seria menos do que proporcional ao aumento efetuado nas tarifas. 


\section{BI BL IOGRAF I A}

BARBOSA, F.H. Microeconomia: teoria, modelos econométricos e aplicaçöes à economia brasileira. IPEA, 1985.

BECKER, G. Economic theory - Alfred Knopf New York - 1971.

CARTTER, A. Theory of wages and employment - Home Wood - 1959.

DAVIS, B.E. \& CHAUDRY. An econometric planning model for the American telephone and telegraph company - Model of ATT - 1975.

FERREIRA, J.L.N. A estrutura tarifária sob regime de monopólio em telecomunica cōes - EMIBRATEL - COM . 3 - 1979.

HARBERGER, A.C. Marginal cost pricing and social investment criteria for electricity undertaking - UNIVERSITY OF CHICAGO, 1964.

HARBERGER, A. \& ANDREATTA, N. A note on the economic principles of electricity pricing. Applied Economics Papers, march, 1963.

JORGENSON, D.W. Econometric studies of investiment behavior a survey - Journal of Economic Literature, 1971.

MITCHELL, Bridger. Optimal pricing of local telephone service. The American Economic Review - September, 1978.

PACEY, P. \& SINGELL, L. Local measured Telephone Service in the USA - Telecommunications Policy - September, 1984.

ROBINSON, J. The economics of imperfect competition - Londres, Macmillan 1933.

RUSSEL, R.R. On the demand curve for a factor of production. American Economic Review - september - 1984.

SCHULTZ, H. The theory and measurements of demand - UNIVERSITY OF CHICAGO 1938.

STIGLER, G. Theory of price. Macmillan, New York, 1966. 